\title{
Preface: Micro- and macroevolution of fishes
}

\author{
Mutsumi Nishida $\cdot$ Koji Maekawa
}

Received: 25 January 2012 / Accepted: 26 March 2012 /Published online: 19 April 2012

(C) Springer Science+Business Media B.V. 2012

Our understanding of evolution has been greatly advanced in the last decade, thanks to the development of molecular genetic techniques for examining genes and the actions of genes in individuals, populations, and even distantly related lineages. Fishes are a huge assemblage of animals comprising more than half of all vertebrate species, having evolved in a wide range of aquatic environments. As such, fishes have contributed greatly to our recent advances in evolutionary studies.

The front line of current evolutionary studies of fishes is growing quickly. In the field of microevolution changes within and among populations, intensive genetic analyses of populations using modern molecular techniques are disclosing the level and pattern of genetic differentiation among populations, enabling us to estimate gene flow among them. Increasing efforts to measure natural selection and fitness in wild populations, using improved statistical analyses,

\section{Nishida}

Department of Marine Bioscience, Atmosphere and Ocean

Research Institute, The University of Tokyo,

5-1-5 Kashiwanoha,

Kashiwa-shi, Chiba 277-8564, Japan

K. Maekawa $(\bowtie)$

Field Science Center for Northern Biosphere,

Hokkaido University,

Nishi 10, Kita 11, Kita-ku,

Sapporo, Hokkaido 060-0811, Japan

e-mail: mkoji@fsc.hokudai.ac.jp are also important for estimating the evolutionary process of ecological and morphological traits in fishes. Macroevolutionary studies have also been promoted by modern molecular techniques, in particular efficient DNA-sequencing technologies. The development of molecular phylogenetic analyses has provided phylogenetic trees for various groups of closely- and distantly-related fishes. In turn such trees have been useful as reliable frameworks for estimating evolutionary patterns of organismal or ecological traits and genes or genomes.

As the front line of research rapidly extends, it is important to have an overview of the present state of evolutionary studies so we can identify the future direction of research. This is why we planned the international symposium on the "Micro- and Macroevolution of Fishes". The aim of the symposium was to bring together fish evolutionary biologists with various backgrounds, from molecular biology and genomics to ecology and systematics, to share their research results and opinions. The symposium was held on 4-5 October 2007, in conjunction with the 40th memorial annual meeting of the Ichthyological Society of Japan in Sapporo, Hokkaido, with the support of the University of Hokkaido (Fig. 1). Two 21st Century Center of Excellence (COE) programs in Hokkaido University ("Neo-Science of Natural History") and University of Tokyo ("Biodiversity and Ecosystem Restoration") co-hosted this international symposium. 
Fig. 1 Group photo of participants in the international symposium on the "Micro- and Macroevolution of Fishes" held on 4-5 October 2007, in conjunction with the 40th annual meeting of the Ichthyological Society of Japan in Sapporo, Hokkaido (photograph by Mutsumi Nishida)

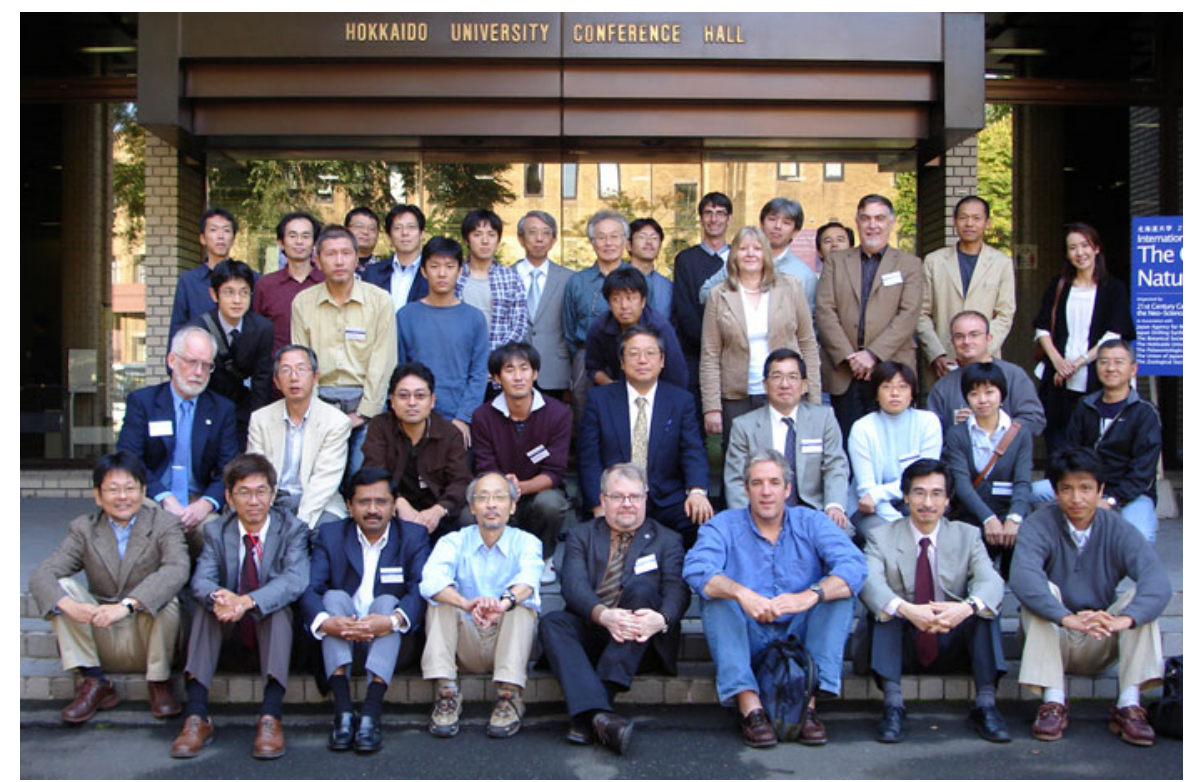

This is a compilation of articles generated mostly from discussions in the symposium. We would like to thank all of the participants who attended the symposium and all of the authors who submitted manuscripts for this compilation in Environmental Biology of Fishes. We are most grateful to David L. G. Noakes, the Editor-in-Chief of the journal, and Lynn Bouvier, the managing editor, for their continued support and assistance. It is our hope that the symposium and this compilation will serve as a stepping-stone to further promotion of active interactions among fish biologists studying microevolution and macroevolution. 